\title{
Clostridium spiroforme
}

National Cancer Institute

\section{Source}

National Cancer Institute. Clostridium spiroforme. NCI Thesaurus. Code C122266.

A species of helically coiled, oblig ately anaerobic, non-motile, gram-positive, spore-

forming, rod-shaped bacteria in the family Clostridiaceae. Unlike most clostridia, C.

spiroforme does not produce butyric acid as an end product of glucose fermentation.

The species produces a toxin that is associated with severe diarrhea and enterocolitis in rabbits. 